\title{
Functional development of fronto-striato-parietal networks associated with time perception
}

\author{
Anna B. Smith ${ }^{1 *}$, Vincent Giampietro ${ }^{2}$, Michael Brammer ${ }^{2}$, Rozmin Halari $^{1}$, Andrew Simmons ${ }^{2,3,4}$ and \\ Katya Rubia ${ }^{1}$ \\ ' Department of Child and Adolescent Psychiatry, Institute of Psychiatry, Kings College London, London, UK \\ ${ }^{2}$ Department of Neuroimaging, Institute of Psychiatry, London, UK \\ ${ }^{3}$ Center for Neurodegeneration Research, Institute of Psychiatry, King's College London, London, UK \\ ${ }^{4}$ NIHR Biomedical Research Centre for Mental Health at South London and Maudsley NHS Trust and Institute of Psychiatry, King's College London, London, UK
}

\section{Edited by:}

Srikantan S. Nagarajan, University of

California San Francisco, USA

Reviewed by:

David T. Blake, Medical College of Georgia, USA

Marc Wittmann, Institute for Frontier Areas of Psychology and Mental

Health, Germany

${ }^{*}$ Correspondence:

Anna B. Smith, Institute of Psychiatry, Box P046, De Crespigny Park, London SE5 8AF, UK.

e-mail:anna.smith@kcl.ac.uk
Compared to our understanding of the functional maturation of executive functions, little is known about the neurofunctional development of perceptive functions. Time perception develops during late adolescence, underpinning many functions including motor and verbal processing, as well as late maturing higher order cognitive skills such as forward planning and future-related decision making. Nothing, however, is known about the neurofunctional changes associated with time perception from childhood to adulthood. Using functional magnetic resonance imaging we explored the effects of age on the brain activation and functional connectivity of 32 male participants from 10 to 53 years of age during a time discrimination task that required the discrimination of temporal intervals of seconds differing by several hundred milliseconds. Increasing development was associated with progressive activation increases within left lateralized dorsolateral and inferior fronto-parieto-striato-thalamic brain regions. Furthermore, despite comparable task performance, adults showed increased functional connectivity between inferior/dorsolateral interhemispheric fronto-frontal activation as well as between inferior fronto-parietal regions compared with adolescents. Activation in caudate, specifically, was associated with both increasing age and better temporal discrimination. Progressive decreases in activation with age were observed in ventromedial prefrontal cortex, limbic regions, and cerebellum. The findings demonstrate age-dependent developmentally dissociated neural networks for time discrimination. With increasing age there is progressive recruitment of later maturing left hemispheric and lateralized fronto-parieto-striato-thalamic networks, known to mediate time discrimination in adults, while earlier developing brain regions such as ventromedial prefrontal cortex, limbic and paralimbic areas, and cerebellum subserve fine-temporal processing functions in children and adolescents.

Keywords: development, time discrimination, functional magnetic resonance imaging

\section{INTRODUCTION}

Temporal perception of relatively brief durations in the range of seconds and milliseconds is integral to motor and verbal function as well as speed of cognitive processing. Just as motor skills (Rueckriegel et al., 2008), verbal tempo (Kowal et al., 1975), and speed of cognition (Hale, 1990) continue to develop throughout adolescence into early adulthood, so do temporal processing skills within this temporal range, including accuracy of motor tapping (McAuley et al., 2006), the detection of very brief temporal gaps (Fischer and Hartnegg, 2004), time discrimination, and perception (Drake et al., 2000) as well as other temporal processing functions (Dawes and Bishop, 2008). Different functional operations within short time ranges of a few seconds and milliseconds are thought to use a common mechanism (Keele et al., 1985; Ivry and Hazeltine, 1995). There is evidence that temporal processes in different time domains are closely interrelated and thus developmental changes in temporal processing abilities in the milliseconds temporal range may be associated with developmental changes in other temporal processing skills. These include the ability to process longer durations of several seconds or more (Rozek et al., 1977; Block et al., 1999; Droit-Volet and Wearden, 2001; Szelag et al., 2002) as well as other cognitive functions requiring temporal processing in other time domains such as temporal discounting, requiring temporal foresight (Steinberg et al., 2009), all skills which will contribute to an extended and deeper temporal perspective in late adolescence (Greene, 1986).

Despite time perception developing late during adolescence, no study has yet investigated the neurofunctional maturational changes underlying this important process between childhood and adulthood. The majority of developmental functional imaging studies have focused upon late developing executive functions, showing linear progressive age-associated increases in activation in frontal, striatal, and parietal brain regions during tasks of cognitive control (Rubia et al., 2000, 2006, 2007, 2010; Adleman et al., 2002; Bunge et al., 2002; Booth et al., 2003; Konrad et al., 2005; Marsh et al., 2006; Christakou et al., 2009b, 2011; Smith et al., 2011). 
Some studies report developmental changes within the prefrontal cortex, in either loci (Luna et al., 2001; Adleman et al., 2002; Tamm et al., 2002; Konrad et al., 2005; Rubia et al., 2010) or lateralization, with several studies showing progressive left-lateralization with age within frontal brain regions (Rubia et al., 2000; Bunge et al., 2002; Marsh et al., 2006; Christakou et al., 2011).

These progressive developmental changes appear to be coupled with progressive activation decreases in earlier developing posterior (Rubia et al., 2000, 2006, 2007; Adleman et al., 2002; Bunge et al., 2002; Konrad et al., 2005; Christakou et al., 2009b), limbic, and paralimbic brain regions (Booth et al., 2003; Monk et al., 2003; Marsh et al., 2006; Christakou et al., 2011; Smith et al., 2011). These findings are congruent with structural imaging studies where frontal, parietal (Sowell et al., 2003), and striatal (Sowell et al., 1999) gray and white matter changes are particularly marked during adolescence well into adulthood, while there is early maturation of phylogenetically older regions associated with more basic cognitive and sensory processing, such as posterior, primary sensory, sensori-motor, and limbic regions (Sowell et al., 2003).

Few studies have focused upon the developmental age effects on perceptive functions. We have previously investigated neurofunctional maturation of perceptive attention allocation from childhood to adulthood, finding progressively increased activation with age in lateral inferior fronto-striatal and temporo-parietal brain regions (Rubia et al., 2010). However, no study has as yet investigated the neurofunctional maturation of time perception between childhood and adulthood.

Fine-temporal perception has effectively been measured in fMRI studies using tasks of temporal discrimination of short durations, such as durations which differ by several hundred milliseconds. These studies have demonstrated consistent activation during temporal discrimination in several brain regions that are considered key structures involved within a hypothesized internal clock (Rubia, 2006). These include inferior frontal gyrus (IFG; Maquet et al., 1996; Pedersen et al., 2000; Ferrandez et al., 2003; Smith et al., 2003; Livesey et al., 2007) dorsolateral prefrontal cortex (DLPFC; Maquet et al., 1996; Rao et al., 2001; Nenadic et al., 2003; Smith et al., 2003; Tregellas et al., 2006; Morillon et al., 2009; Shih et al., 2009) the supplementary motor area (SMA; Rao et al., 2001; Ferrandez et al., 2003; Smith et al., 2003; Macar et al., 2006; Tregellas et al., 2006; Morillon et al., 2009; Shih et al., 2009), the insula (Ferrandez et al., 2003; Nenadic et al., 2003; Tregellas et al., 2006; Livesey et al., 2007), the basal ganglia (Dupont et al., 1993; Jueptner et al., 1995; Rao et al., 2001; Ferrandez et al., 2003; Nenadic et al., 2003; Tregellas et al., 2006; Livesey et al., 2007; Shih et al., 2009), and the cerebellum (Dupont et al., 1993; Jueptner et al., 1995; Maquet et al., 1996; Rao et al., 2001; Smith et al., 2003; Mathiak et al., 2004; Tregellas et al., 2006).

A study controlling for the confound of increased task demand, typically found in time discrimination relative to control conditions, showed activation within insula, IFG, and striatum to be specifically associated with time perception (Livesey et al., 2007). The striatum is thought to play a critical computational role in time perception (Grondin, 2010) while frontal regions are thought to mediate between cognition and time perception (Rubia and Smith, 2004; Rubia, 2006; Wittmann et al., 2010).
Other regions found to be activated in tasks of time perception and discrimination such as the anterior cingulate cortex (ACC; Maquet et al., 1996; Nenadic et al., 2003), and the parietal lobes (Dupont et al., 1993; Maquet et al., 1996; Pedersen et al., 2000; Rao et al., 2001; Mathiak et al., 2004; Macar et al., 2006) are thought to have a more generic attentional role, mediating perceptive attention to time intervals.

Only one fMRI study has investigated age effects in a temporal discrimination task, but this was restricted to a group of children and adolescents aged between 10 and 15 years. No developmental change in activation or in functional inter-regional connectivity was observed within this age group (Neufang et al., 2008).

In order to investigate the functional development of finetemporal discrimination between late childhood and adulthood, we used fMRI in 32 male participants between 10 and 53 years old while they performed a time discrimination task that required the discrimination of brief intervals of $1-1.5 \mathrm{~s}$, differing by several hundreds of milliseconds.

Based on previous developmental fMRI studies of executive and attention functions showing progressively increased lateral frontostriatal and fronto-parietal activation with increasing age (Adleman et al., 2002; Bunge et al., 2002; Konrad et al., 2005; Rubia et al., 2006, 2007, 2010; Christakou et al., 2009b, 2011; Smith et al., 2011), and evidence for the importance of lateral inferior and dorsolateral fronto-insular-striatal networks for temporal discrimination in short temporal range of milliseconds and seconds (Matell and Meck, 2004; Rubia and Smith, 2004; Coull et al., 2011), we hypothesized that increasing age between childhood and mid-adulthood would be associated with a linear increase in activation in these key areas of time discrimination. Furthermore, given evidence for reduced functional connectivity in children compared to adults in the context of other cognitive tasks (Rubia et al., 2007; Stevens et al., 2007; Christakou et al., 2011) we hypothesized that children would show reduced functional inter-regional connectivity in key fronto-striatal areas of time discrimination compared to adults.

\section{MATERIALS AND METHODS SUBJECTS}

Thirty-two right-handed male volunteers took part in the study, including 17 right-handed boys and 15 right-handed men. The mean age of the total sample was 21 years (SD 7 years and 6 months) and subjects ranged from 10 to 53 years of age. The mean age of adults was 29 years and 8 months (SD: 8 months) ranging from 10 to 16 while the mean age of children and adolescents was 14 years (SD: 2 months) ranging from 20 to 53 .

All subjects achieved a score above the 10th percentile on the Ravens Standard Progressive Matrices (a non-verbal test of IQ; Raven et al., 1998).

Exclusion criteria were current or past substance abuse, head injury, mental retardation, or mental or neurological disorder.

All participants were paid $\mathfrak{E} 22$ for participating and written informed consent/assent and approval from the local Ethical Committee was obtained.

There was a significant correlation between age and IQ $(r=0.5$; $p=0.005$ ), and significant between-group differences in IQ scores between the two age groups (Converted mean IQ estimate: adults: 
118 (16); adolescents: $103(14) ; t=0.2 .8 ; \mathrm{df}=28 ; p=0.01$ ). Given these significant effects, IQ was included as a covariate in both the performance and neurofunctional analyses.

\section{TIME DISCRIMINATION TASK}

The time discrimination task consisted of $5 \times 30$-s blocks for each of two conditions, a time discrimination task and a temporal order judgment control condition. Each block began with a 3$s$ instruction period followed by six trials of 4.4-4.6 s, including the presentation of the durations and $2.1 \mathrm{~s}$ for response time. The blocks were alternated, starting with the control condition first. The time discrimination condition, was indicated by the appearance of the letter "L" denoting the question "which circle lasts the longest?" for $3 \mathrm{~s}$ in a gray circle of $5 \mathrm{~cm}$ diameter at the center of the screen at the beginning of the block. The subject was then presented with a red and green circle, $5 \mathrm{~cm}$ in diameter, appearing consecutively with no intermittent pause and in random order. The red circle was consistently presented on the left side and the green circle consistently on the right side of the center of the computer screen, with a gap of $1 \mathrm{~cm}$ separating their positions. One circle was randomly presented for $1000 \mathrm{~ms}$, and the comparison circle for either 1300,1400 , or $1500 \mathrm{~ms}$. The subjects held a response box for the duration of the task and were told beforehand that in the experimental condition indicated by the letter "L" they had to decide which of the two circles stayed on the screen for the "Longest" time. They were told to respond using a left-sided button if the red circle, displayed on the left side of the screen, lasted longest, or a right-sided button if the green circle, displayed on the right side, lasted longest. Thus a block of trials consisted of two trials each of the following: 1000 versus $1300 \mathrm{~ms} ; 1000$ versus $1400 \mathrm{~ms}$; and 1000 versus $1500 \mathrm{~ms}$, with $2100 \mathrm{~ms}$ allocated for response time.

The temporal order judgment (control) condition was identical to the time discrimination condition, with duplicate trials of green and red circles of different durations presented in exactly the same way. The only difference was that these blocks began with the presentation of the number "2" (denoting the question "which circle comes second?") for $3 \mathrm{~s}$ displayed in a gray circle at the center of the screen. These trials required that, rather than attending to the duration of the circles, the subject had to attend to the order of appearance of the circles and indicate which circle came second using the same response buttons in the way described for the time discrimination condition. The task was visually presented to the subjects in the MRI scanner via a prism from a liquid crystal diode projector.

\section{TASK PERFORMANCE ANALYSIS}

Both accuracy of order judgment and accuracy of time discrimination as measured by number of errors were analyzed by (a) two-way analysis of variance (ANCOVA) with one between subjects factor of age group (children and adolescents; adults) and one within-subjects factor of comparison duration length (1300; 1400; $1500 \mathrm{~ms}$ ) and with IQ as a covariate and (b) correlation between age and time estimation errors and correlation between age and order judgment errors (controlling for IQ).

\section{fMRI DATA ANALYSIS}

During the task, functional images were acquired on a $1.5-\mathrm{T}$ GE Neuro-optimized Signa LX Horizon System (General Electric,
Milwaukee, WI, USA), using a gradient echo planar sequence sensitive to blood oxygenation level dependent (BOLD) contrast. In each of 16 non-contiguous planes parallel to the anteriorposterior commissure, $100 \mathrm{~T} 2^{*}$-weighted MR images depicting BOLD contrast were acquired with $\mathrm{TE}=40 \mathrm{~ms}$; $\mathrm{TR}=3$-s; flip angle $90^{\circ} ; 64 \times 64$ matrix; in-plane voxel size $3.75 \mathrm{~mm} \times 3.75 \mathrm{~mm}$; slice thickness $=7 \mathrm{~mm}$, slice-skip $=0.7 \mathrm{~mm}$. A birdcage head coil was used for RF transmission and reception. Head movement was limited by foam padding within the head coil and a restraining band across the forehead. Quality control was carried out using an automated analysis tool to ensure high quality images (Simmons et al., 1999).

The fMRI data were analyzed with the XBAM software developed at the Institute of Psychiatry (c.f. http://brainmap.it). This method of analysis makes no normality assumptions, which are usually violated in fMRI data, but instead uses median statistics to control outlier effects and permutation rather than normal theory based inference. Furthermore the most common test statistic is computed by standardizing for individual difference in residual noise before embarking on second level, multi-subject testing using robust permutation-based methods. This allows a mixed effects approach to analysis - an approach that has recently been recommended following a detailed analysis of the validity and impact of normal theory based inference in fMRI in large number of subjects (Thirion et al., 2007).

\section{Individual analysis}

Functional magnetic resonance imaging data were realigned to minimize motion-related artifacts (Bullmore et al., 1999a), and smoothed using a Gaussian filter (full-width half maximum, $7.2 \mathrm{~mm}$ ). Time series analysis of individual subject activation was performed using wavelet-based re-sampling method previously described (Bullmore et al., 2001). Briefly, we first convolved each experimental condition with two Poisson model functions (delays of 4 and $8 \mathrm{~s}$ ). We then calculated the weighted sum of these two convolutions that gave the best fit (least-squares) to the time series at each voxel. A goodness-of-fit statistic (the SSQ-ratio) was then computed at each voxel consisting of the ratio of the sum of squares of deviations from the mean intensity value due to the model (fitted time series) divided by the sum of squares due to the residuals (original time series minus model time series). The appropriate null distribution for assessing significance of any given SSQ-ratio was established using the wavelet-based data re-sampling method (Bullmore et al., 2001) and applying the model-fitting process to the re-sampled data. This process was repeated 20 times at each voxel and the data combined over all voxels, resulting in 20 null parametric maps of SSQ-ratio for each subject, which were combined to give the overall null distribution of SSQ-ratio. The same permutation strategy was applied at each voxel to preserve spatial correlation structure in the data. Activated voxels, at a $<1$ level of Type I error, were identified through the appropriate critical value of the SSQ-ratio from the null distribution. Individual maps were registered into Talairach space using rigid body and affine transformation.

\section{Group analysis}

A generic group activation map was then produced for this task. Individual SSQ-ratio maps were transformed into standard 
space, first by rigid body transformation of the fMRI data into a high-resolution inversion recovery image of the same subject, and then by affine transformation onto a Talairach template. White matter regions were extracted from our analysis using the BET tool from the FSL software package (Smith, 2002). This creates a gray matter mask of the Talairach template used for normalization. This mask was subsequently used to restrict the analysis to those voxels lying within gray matter.

A generic activation group map was produced for the experimental condition (time discrimination-temporal order) by calculating the median observed SSQ-ratio over all subjects at each voxel in standard space and testing them against the null distribution of median SSQ-ratios computed from the identically transformed wavelet re-sampled data (Brammer et al., 1997). The voxel level threshold was first set to 0.05 to give maximum sensitivity and to avoid Type II errors. Next, a cluster level threshold was computed for the resulting 3-D voxel clusters such that the final expected number of Type I error clusters was $<1$ per whole-brain. The necessary combination of voxel and cluster level thresholds was not assumed from theory but rather was determined by direct permutation for each data set, giving excellent Type II error control (Bullmore et al., 1999b). Instead of relying on asymptotic distributions such as $t$ or $F$ that assume data normality, we use data-driven, permutation-based methods with minimal distributional assumptions that have been shown to be more suitable for fMRI data analysis in these kind of cluster sizes (Zhang et al., 2009). Cluster mass rather than a cluster extent threshold was used to minimize discrimination against possible small, strongly responding foci of activation (Bullmore et al., 1999b). Briefly, a voxel-wide significance threshold was set $(p<0.05)$, and surviving voxels were assembled into 3 -D clusters using a contiguity criterion. The mass of each cluster was calculated by adding the statistical values of all cluster members and thresholded at $p<0.03$. Thus, less than one false positive activation locus was expected for $p<0.05$ at voxel level and $p<0.03$ at cluster level.

\section{Whole-brain correlations between brain activation and age across all subjects}

A whole-brain linear correlation was computed between age and brain activation for the contrast of time discrimination and temporal order, while covarying for IQ, in order to take into account the significant correlation of IQ with age. Firstly, the Pearson product-moment correlation coefficient was computed at each voxel in standard space between age data and signal change over all subjects. The correlation coefficients were recalculated after randomly permuting the age values between subjects. Repeating the second step many times (1,000 times per voxel, then combining over all voxels) gives the distribution of correlation coefficients under the null hypothesis that there is no association between specific ages and specific BOLD effects. This null distribution can then be used to assess the probability of any particular correlation coefficient under the null hypothesis. The critical value of the correlation coefficient at any desired Type I error level in the original (non-permuted) data was determined by reference to this distribution. Statistical analysis was extended to cluster level as described previously (Bullmore et al., 1999b). Thus, less than one false positive activation locus was expected for $p<0.05$ at voxel level and $p<0.05$ at cluster level.
For all experimental contrasts, information can be obtained about the size and direction of the activation from the general linear model fit to the time series of activation. The sign of the BOLD response can either be positive or negative with respect to the regressor. It is possible that activation clusters that correlate positively with age are brain regions that correlated negatively with the order judgment control task, or that brain clusters that correlated negatively with age were brain clusters that increased with age during the order judgment control condition. In order to be sure that the positive and negative associations with age were related to activation clusters that were associated with time discrimination we examined the sign of the signal change relative to the regressor and BOLD responses were only considered where the average SSQ-ratio in response to the activation condition was positive (i.e., associated with time discrimination versus order judgment) and where the positive activation changed positively or negatively with age.

In order to examine whether clusters of activation which were significantly correlated with age were associated with performance, standardized BOLD response values (SSQ-ratios) were extracted from each cluster. Pearson's product-moment correlation coefficients were calculated for these sets of values and performance as measured by accuracy of time discrimination.

\section{Whole-brain correlations between brain activation and performance across all subjects}

A whole-brain linear correlation was computed between performance as measured by percentage of correct time discrimination trials and brain activation for the contrast of time discrimination and temporal order. Although there was no correlation of IQ with time discrimination performance, we covaried for IQ in order to obtain clusters which were directly related to time discrimination performance. Firstly, the Pearson product-moment correlation coefficient was computed at each voxel in standard space between performance data and signal change over all subjects. The correlation coefficients were recalculated after randomly permuting the performance values between subjects. Repeating the second step many times (1,000 times per voxel, then combining over all voxels) gives the distribution of correlation coefficients under the null hypothesis that there is no association between performance and specific BOLD effects. This null distribution can then be used to assess the probability of any particular correlation coefficient under the null hypothesis. The critical value of the correlation coefficient at any desired Type I error level in the original (non-permuted) data could be determined by reference to this distribution. Statistical analysis was extended to cluster level as described previously (Bullmore et al., 1999b). Thus, less than one false positive activation locus was expected for $p<0.05$ at voxel level and $p<0.05$ at cluster level.

\section{Conjunction analysis to find overlapping regions which increase in activation for age and performance}

To identify overlapping brain activation patterns associated with both increasing age and with accuracy, we used a random-effects conjunction analysis, based on inclusive masking (Nichols et al., 2005) on two brain maps: These maps included activation resulting from the whole-brain correlation analyses between age and activation, showing positive activation increase with age (see 
Table 1); and a second map resulting from the whole-brain correlation analysis between performance and activation, showing positive activation increase with performance as measured by percentage of correct responses (see Table 1). This conservative analysis is based upon a logical AND operation, mapping those voxels which are significantly associated with both accuracy of timing and increase in age. We used a threshold of $p<0.05$, corrected for multiple comparisons at the voxel level.

\section{Whole-brain correlations between brain activation and IQ across all subjects}

In order to report the independent effect of IQ on time discrimination, a whole-brain linear correlation was computed between IQ and brain activation for the contrast of time discrimination and temporal order as described above.

\section{Analysis of functional connectivity in task-relevant brain regions}

In order to test for group differences in functional connectivity between children and adults, regions of interest (ROIs) were identified on the basis of the three clusters identified as significant across all subjects for the contrast of time discrimination versus order blocks ( $p<0.05$ at voxel, and $p<0.03$ at cluster levels; see Table 1). The sample was then divided into child/adolescent and adult age groups as defined above. Average time series were then extracted for each group within individual clusters and corrected for motion and timing differences in slice acquisition. Pearson correlation coefficients between each ROI pair were then compared across groups following Fisher's $z$ transformation. The probability threshold for significance was corrected for multiple testing using the Dunn-Sidak method.

\section{RESULTS}

\section{TASK PERFORMANCE}

The correlational analysis, controlling for IQ, showed no significant linear associations between age and error rates for time discrimination $(r=0.16 ; p=0.42)$ or order judgment $(r=0.04$; $p=0.85$ ). An ANCOVA with IQ as covariate between children and adults showed no significant differences for each age group in error rates $(F=1.7 ; \mathrm{df}=30 ; p=0.20)$, no effect of comparison duration $(F=0.5 ; p=0.58)$, and no interaction of group and duration comparison $[F=0.7 ; p=0.4$; see Figure 1 ; mean $(\mathrm{SD})$ percentage errors was 12.2 (13.5) for adults and 15.9 (10.5) for adolescents]. No significant effect of IQ was observed ( $F=0.047 ; p=0.83)$. An ANCOVA with IQ as a covariate between children and adults also showed no significant group differences in error rates for order judgment $[F=0.32 ; p=0.72$; mean (SD) percentage errors was 3.6 (7.6) for adults and 5.3 (12.8) for adolescents]. No significant effect of IQ was observed $(F=0.73 ; p=0.40)$.

\section{BRAIN ACTIVATION Head motion}

No subject demonstrated head motion exceeding a cut-off of 1 voxel (maximum value of $x$ : 0.29 ; max value of $y: 0.21$; max value of $z: 0.3$ ) and no significant correlations were observed between age and mean head motion. A MANOVA testing differences between the largest displacement translational value in adolescents and adults for head motion across the $x, y$, and $z$ axis was not significant.

\section{Significant activation across the whole group}

Three significant clusters of activation were observed for the contrast of time discrimination blocks versus order blocks (see Table $\mathbf{1}$;

Table 1 | Clusters of significant activation for all subjects during contrast of temporal discrimination and order as well as the results of a whole-brain regression analysis showing brain areas where activation has positive and negative linear correlation with age during time discrimination contrast with $I Q$ as a covariate.

\begin{tabular}{|c|c|c|c|c|c|c|}
\hline Size & \multicolumn{3}{|c|}{ Talairach coordinates } & $\begin{array}{l}p \text { value } \\
<=\end{array}$ & BA & Region \\
\hline 97 & -29 & 19 & 9 & 0.0063 & 44 & $L$ inferior frontal gyrus \\
\hline 466 & -4 & 7 & 42 & 0.0011 & $47,24,32$ & $\mathrm{R}$ orbitofrontal cortex, caudate, bilateral anterior cingulate \\
\hline 39 & 47 & -41 & 42 & 0.0147 & 40 & $\mathrm{R}$ inferior parietal lobe \\
\hline 28 & -54 & 30 & 15 & 0.0000 & 46 & L dorsolateral, inferior prefrontal cortex \\
\hline 75 & 29 & 7 & 9 & 0.0000 & $\mathrm{n} / \mathrm{a}$ & R putamen, caudate, thalamus, insula \\
\hline 33 & 11 & -56 & 64 & 0.0000 & 7 & $\mathrm{R}$ precuneus \\
\hline 16 & 18 & -70 & 58 & 0.0250 & 7 & R superior parietal lobe \\
\hline 36 & -25 & -77 & 20 & 0.0000 & 7 & $L$ cuneus \\
\hline 26 & 25 & -78 & 20 & 0.0000 & 18 & $R$ cuneus \\
\hline 94 & -22 & 11 & -24 & 0.0000 & 11,47 & $\mathrm{~L}$ ventromedial frontal gyrus \\
\hline 42 & 14 & -26 & -29 & 0.0000 & $\mathrm{n} / \mathrm{a}$ & $\mathrm{R}$ brain stem: pons \\
\hline 14 & 14 & -44 & 9 & 0.0072 & 29,30 & $\mathrm{R}$ posterior cingulate \\
\hline 22 & 43 & -48 & -35 & 0.0011 & 35,36 & R cerebellum, anterior lobe (culmen), parahippocampal gyrus \\
\hline
\end{tabular}




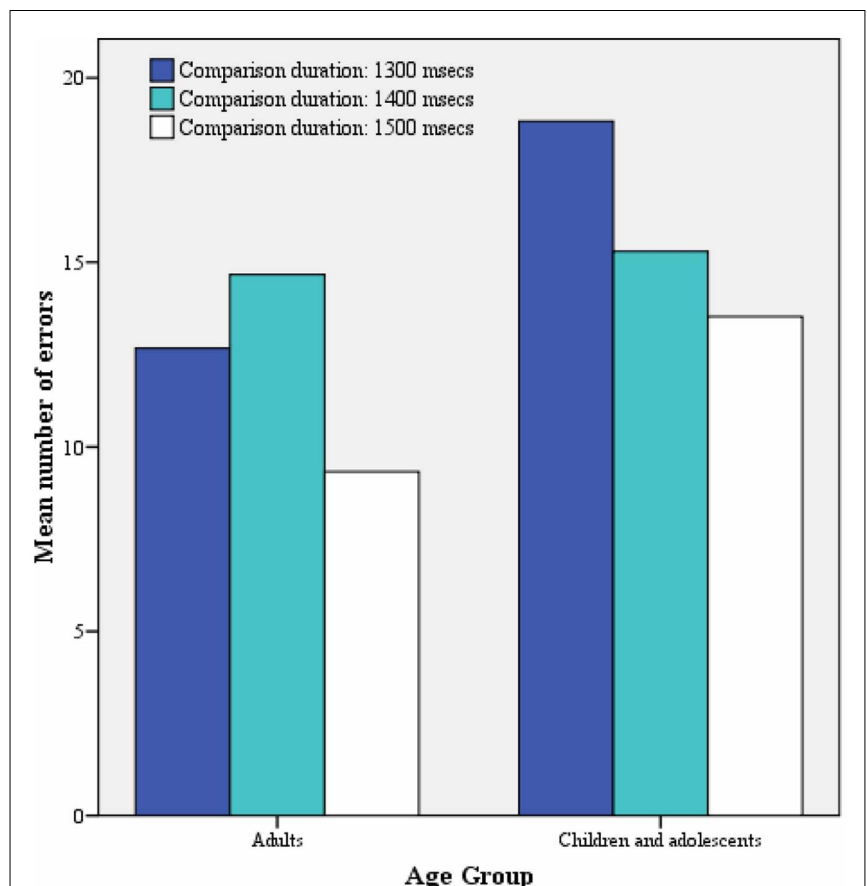

FIGURE 1 | Mean number of time discrimination errors for each comparison duration for each age group.

Figure 2). The first extended medially from left IFC, precentral gyrus, and SMA into caudate, the second extended from right ventral and DLPFC, insula, medial frontal cortex (SMA/ACC) into caudate, and thalamus. The third cluster was located in right inferior parietal lobe.

\section{Whole-brain regression analysis between age and activation}

Activation associated with time discrimination increased significantly with age in left dorsolateral prefrontal and inferior frontal cortices, right dorsal striatum and thalamus and insula, left uncus, right parietal lobe, and bilateral cuneus (see Table 1; Figure 3). Activation decreased significantly with age within left ventromedial and right rostromedial prefrontal cortex, left ACC, right posterior cingulate cortex (PCC), hippocampus, brain stem, and right cerebellum (see Table 1; Figure 3).

\section{Whole-brain regression analysis between performance and activation}

Activation associated with time discrimination increased significantly with performance in right dorso- and ventro-lateral frontal cortex, ACC, and caudate, as well as in more posterior regions including right temporo-parietal cortex and bilateral cerebellum (see Table 2).

\section{Conjunction analysis between whole-brain regression analyses between brain activation and age and activation and performance}

The conjunction analysis between the two whole-brain regression analyses between activation and age and activation and performance described above showed that activation in right caudate was positively correlated with both age and performance (see Figure 3 ).
It is possible that linear changes with age across the age range of 10-43 years may be largely driven by maturational changes in adolescence. Therefore, in order to test whether increases in brain activation with age were disproportionately driven by the younger participants, we used the Fisher $r-z$ transformation to compare the correlation coefficients of the adolescent and adult subgroups for each cluster identified in the whole-brain age-correlation analysis. There were no significant differences in correlation coefficients between the two subgroups, suggesting that the linear changes were not disproportionately associated with adolescence.

Given the significant differences in IQ between the two age groups and the significant correlation with age and IQ, we wanted to ensure that IQ-related activation was not related to activation associated with developmental change. We therefore carried out a whole-brain regression analysis between IQ and activation: Activation during time discrimination was significantly positively correlated with increasing IQ scores in a cluster extending rostrally from PCC through hippocampal gyrus into cerebellum (Talairach coordinates of peak response: $x=14 ; y=-52 ; z=-7)$. A conjunction analysis showed that this cluster did not overlap with any of the significant clusters in the whole-brain regression analyses between age and activation during time discrimination.

\section{Between-group differences in functional connectivity}

For this analysis, the sample was grouped into adolescents and adults: Tests for differences between these two groups in the intercorrelations between the time series for each of the three selected ROIs (areas activated across the whole group for time discrimination - order judgment) showed that compared with adults, adolescents had significantly reduced intercorrelations between the cluster of activation within right ventral prefrontal cortex that reached into insula, caudate and thalamus, and the cluster of activation in right inferior parietal lobe $(z=2.86$; $p=0.004)$. Reduced intercorrelations in adolescents relative to adults were also observed between the same right prefrontal cluster of activation and a homologue cluster of activation within the left hemisphere, comprising left IFC, SMA, and caudate, but were just above the corrected probability threshold of $p=0.016(z=2.37$; $p=0.017$ ).

\section{DISCUSSION}

Across all subjects, time discrimination of second durations differing by several hundred milliseconds was associated with activation within bilateral fronto-striato-parietal regions. Activation specifically associated with time discrimination performance was observed in right IFG, OFC, and ACC, temporo-parietal junction, left caudate, and bilateral cerebellum. Despite no age effects on task performance, the whole-brain regression analysis showed progressive age-associated increases in activation in left dorsolateral/inferior prefrontal cortex, and in right hemispheric striato-thalamic and superior parietal regions. Regressive changes in brain activation with development were observed within early developing midline paralimbic and limbic regions, including left ventromedial and right rostromedial prefrontal cortex, bilateral cingulate, hippocampus, brain stem as well as cerebellum. A conjunction analysis between the two whole-brain regression analyses between activation and (a) age and (b) performance showed that 


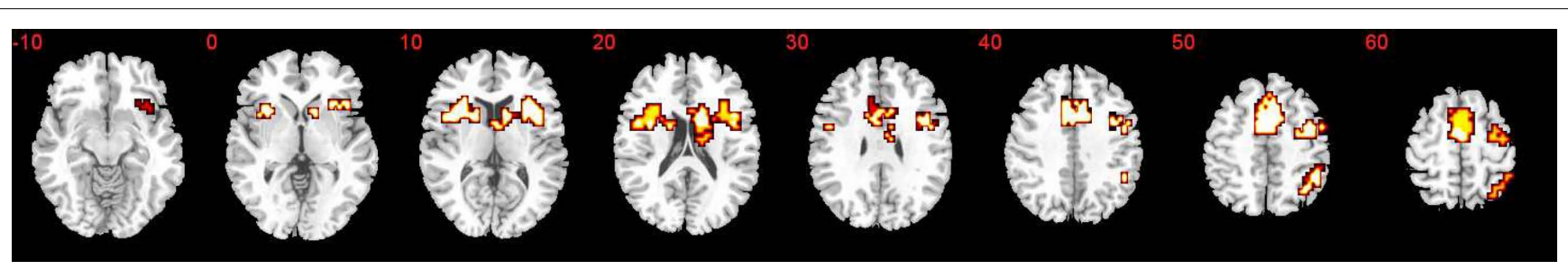

FIGURE 2 | Brain activation during contrast of time discrimination versus temporal order for all subjects. Statistical threshold selected to elicit less than on error cluster ( $p<0.05$ for voxel and $p<0.03$ for cluster-wise analysis). Slices are marked with the $z$ coordinate as distance in millimeters from the anterior-posterior commissure.

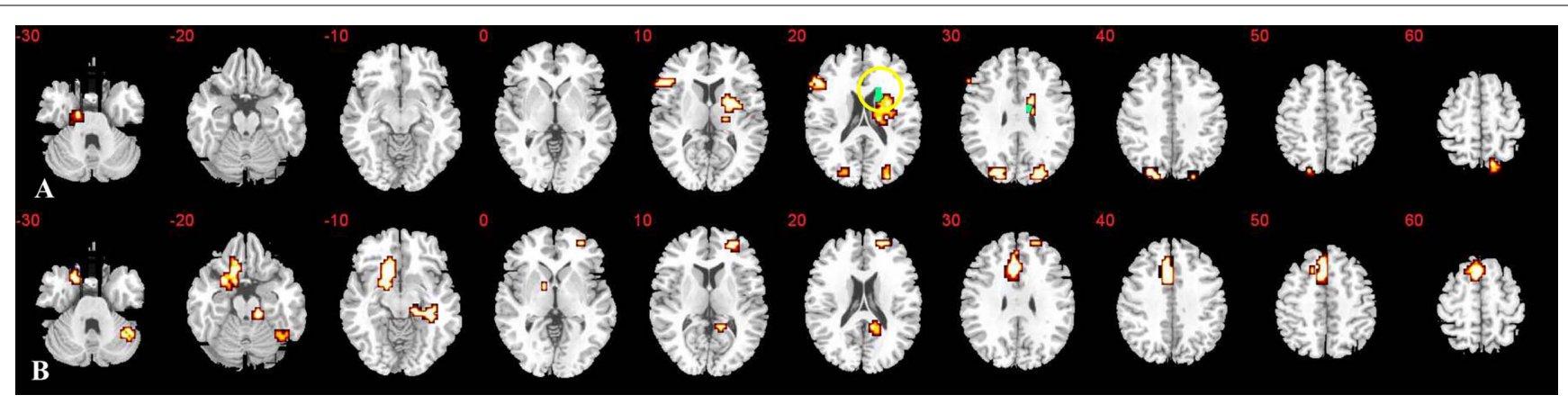

FIGURE 3 | Whole-brain correlation of activation with age: clusters exhibiting linear positive (A) or negative (B) correlation with age across all subjects. Statistical threshold selected to elicit less than one error cluster (at $p<0.05$ for voxel and $p<0.05$ for cluster levels). Slices are marked with the $z$ coordinate as distance in millimeters from the anterior-posterior commissure. Blue regions show caudate activation also directly related to performance as demonstrated by conjunction analysis.

Table 2 | Results of a whole-brain regression analysis showing brain areas where activation has positive correlation with performance during time discrimination contrast with $I Q$ as a covariate.

\begin{tabular}{|c|c|c|c|c|c|}
\hline \multirow[t]{2}{*}{ Size } & \multicolumn{2}{|c|}{ Talairach coordinates } & $p$ value & \multirow[t]{2}{*}{ BA } & \multirow[t]{2}{*}{ Region } \\
\hline & $x$ & $z$ & $<=$ & & \\
\hline
\end{tabular}

\begin{tabular}{|c|c|c|c|c|c|c|}
\hline \multicolumn{7}{|c|}{ POSITIVE CORRELATION WITH PERFORMANCE } \\
\hline 69 & 36 & 30 & 42 & 0.0001 & 47 & $\mathrm{R}$ inferior frontal gyrus \\
\hline 17 & 47 & 30 & -29 & 0.0001 & 47 & $\mathrm{R}$ inferior frontal gyrus \\
\hline 136 & 14 & 22 & 9 & 0.0001 & 9,46 & $\mathrm{R}$ dorsolateral prefrontal cortex \\
\hline 46 & 40 & 11 & -18 & 0.0001 & 47 & $\mathrm{R}$ ventro-lateral prefrontal cortex \\
\hline 58 & 11 & -11 & 31 & 0.0001 & 24 & $\mathrm{R}$ anterior cingulate \\
\hline 203 & -7 & -19 & 20 & 0.0001 & & L caudate \\
\hline 25 & -54 & -26 & 15 & 0.0009 & 40 & L post central gyrus \\
\hline 28 & 61 & -33 & 26 & 0.0001 & 40 & $\mathrm{R}$ inferior parietal lobe \\
\hline 7 & 69 & -37 & 9 & 0.0396 & 42 & R superior temporal gyrus \\
\hline 83 & 4 & -70 & -18 & 0.0001 & & R cerebellum, posterior lobe \\
\hline 17 & -18 & -78 & -35 & 0.0057 & & L cerebellum, posterior lobe \\
\hline 32 & -4 & -78 & 9 & 0.0001 & & L cerebellum, posterior lobe \\
\hline
\end{tabular}

the age-related progressive activation increase in the caudate was specifically associated with increasing accuracy in temporal discrimination. Furthermore, functional connectivity analyses comparing adults and adolescents showed that adults had enhanced functional connectivity between right inferior fronto-striatal and inferior parietal regions and in the interhemispheric connection between left and right inferior frontal cortex. The findings extend previous evidence for progressive fronto-striato-parietal functional brain maturation in the context of tasks of executive functions (Booth et al., 2003; Monk et al., 2003; Marsh et al., 2006; Rubia et al., 2010; Smith et al., 2011) to the domain of a perceptive function of temporal discrimination. Furthermore, the enhanced right inferior fronto-striato-parietal and inferior fronto-frontal interregional connectivity in adults compared with adolescents are 
suggestive of the development of entire neurofunctional networks of time discrimination and not just isolated regions.

Both IFC (Maquet et al., 1996; Pedersen et al., 2000; Ferrandez et al., 2003; Smith et al., 2003; Livesey et al., 2007) and DLPFC (Maquet et al., 1996; Rao et al., 2001; Nenadic et al., 2003; Smith et al., 2003; Tregellas et al., 2006; Morillon et al., 2009; Shih et al., 2009) are key areas of temporal discrimination of intervals of several seconds. Right DLPFC has been posited to have the role of either a central time keeper (Constantinidis et al., 2002; Lewis, 2002) a working memory style accumulator (Mangels et al., 1998; Gruber et al., 2000) or both (Rubia and Smith, 2004). Right IFC has also been shown (along with striatum) to have an unconfounded role in temporal discrimination within this temporal range, given its consistent activation when controlling for difficulty levels of time discrimination (Livesey et al., 2007). We found bilateral inferior prefrontal activation in the group activation map across all ages but specifically left hemispheric DLPFC and IFC activation to increase significantly with age. The lack of a developmental effect in the activation of right DLPFC and IFC suggests that adolescents already activate these right hemispheric regions in response to time discrimination, followed by additional recruitment of the left hemisphere homologue with increasing development. The increase in left hemispheric DLPFC/IFC activation is in line with a recent developmental study of temporal discounting, where left DLPFC activation increased with age during immediate and delayed choices (Christakou et al., 2011). The progressive age-associated recruitment of left hemispheric IFC together with age-unrelated right IFC activation is parallel to structural connectivity findings in adolescence (Blanton et al., 2004) where left, but not right white matter tracts develop with age in 6- to 15-year-old boys. These functional developmental changes may thus reflect more pronounced maturational changes in left hemispheric DLPFC and IFC regions. The enhanced functional connectivity between right and left IFC cortices implies that development is associated with increased interhemispheric communication between left and right IFC during fine-temporal discrimination.

The conjunction analysis showed that caudate activation was associated with both increasing age and better temporal discrimination. This is consistent with evidence that the striatum is specifically involved in mediating temporal discrimination of small intervals of milliseconds and seconds, where its activation has been shown to be independent of task demands (Livesey et al., 2007) and sensory modality (Shih et al., 2009).

The progressively increased superior parietal activation as well as greater functional connectivity between inferior frontal and inferior parietal cortices in adults relative to children is in line with the role of this region in attentional aspects of time discrimination, in particularly sustained attention to time (Pardo et al., 1991; Ortuno et al., 2002; Mathiak et al., 2004), which is impaired in younger children during duration estimations (Gautier and Droit-Volet, 2002).

The findings of increased fronto-striato-parietal networks in older relative to younger subjects extend previous findings of enhanced fronto-parietal and fronto-striato-thalamic interregional connectivity in adults compared to children during executive function tasks such as trail-making, motor inhibition, and temporal discounting (Rubia et al., 2007; Seeley et al., 2007; Christakou et al., 2011). To our knowledge, this is the first study to show that prefrontal, basal ganglia, and parietal regions increase progressively with age in their inter-regional functional communication during a perceptive task.

Younger people, on the other hand, seem to rely upon earlier developing and less specialized limbic, paralimbic, and posterior brain regions for time perception: regressive age-associated effects were observed in ventromedial prefrontal cortex, anterior and PCC, hippocampus, brain stem, and right cerebellum.

As part of the extended limbic system, the ACC has previously been implicated in temporal discrimination of brief intervals (Maquet et al., 1996; Lejeune et al., 1997; Nenadic et al., 2003). However, rather than having a central time-keeping role (Nenadic et al., 2003; Rubia and Smith, 2004), the ACC also is thought to have a more generic role involving selective, and output-related, executive attention (Ullsperger and van Cramon, 2001; Rubia et al., 2003, 2006, 2007; Ridderinkhof et al., 2004). In line with this, ACC activation has been associated with conditions where motor output is required during tasks loading heavily on temporal unpredictability, requiring a higher load on selective and motor attention (Pardo et al., 1991; Paus et al., 1993; Devinsky et al., 1995) including time discrimination (Maquet et al., 1996). Likewise, the progressive PCC activation decrease with age may be related to a more generic role of this region in visual-spatial attention allocation (Lark et al., 2000; Kiehl et al., 2001; Mesulam et al., 2001; Ardekani et al., 2002; Small et al., 2003; Gur et al., 2007; Mohanty et al., 2008) which is a necessary basis function to discriminate two visually displayed time intervals and which may have been recruited more by children due to enhanced effort in the visual-spatial discrimination of time intervals.

Left ventromedial orbitofrontal cortex (vmOFC) activation was also negatively correlated with age: this region is thought to have an important role as a comparator, storing, and utilizing information over brief time delays (Floresco et al., 1997; Rogers et al., 2004), and holding information in representative memory (Schoenbaum et al., 2006), functions that underpin time discrimination of brief durations. Furthermore, the vmOFC is also a key region for decision making (Christakou et al., 2009a, 2011; Lawrence et al., 2009) which may have been more difficult for children than adults. Our findings of more ventromedial FL activation in younger participants extends evidence for recruitment of this region in 8- to 15-year-old children (Neufang et al., 2008), but not adults during the same task (Smith et al., 2003). Children appear thus to rely more on earlier developing ventromedial frontal brain regions for time perception, while with increasing age there is a shift to recruitment of more task-relevant left hemispheric lateral inferior prefrontal regions.

The regressive age-associated changes in the cerebellum are interesting. The importance of the cerebellum in fine-temporal processes within the milliseconds range is fairly well established (Braitenberg, 1967; Harrington and Haaland, 1999), presumably as part of fronto-cerebellar neural networks for time estimation (Ivry and Keele, 1988; Ivry and Diener, 1991; Rao et al., 1997; Penhune et al., 1998; Justus and Ivry, 2001; Rubia, 2006), regardless of difficulty level (Tregellas et al., 2006). The regressive age-associated findings for the cerebellum, together with the 
progressive age-associated changes in prefrontal regions, therefore suggests that adults rely more on frontal parts of this frontocerebellar neural network of fine-temporal perception, while children activate more posterior cerebellar parts of this network.

In conclusion, it appears that younger participants activate to a greater extent, earlier maturing midline frontal, limbic, paralimbic (Joseph, 1999), and posterior brain regions (Chugani et al., 2008), which appear to mediate more generic attention functions underlying time perception. With increasing maturity, however, time discrimination appears to be become progressively more mediated by the activation as well as functional connectivity of more focal, specialized lateral fronto-striatal, and parietal regions which are known to mature later in their brain structure (Sowell et al., 1999; Gogtay et al., 2004).

We did not observe any significant performance differences between the two age groups, although children had slightly larger error rates. This could be due to the possibility that younger participants used different strategies and associated networks to do the task, which they may have found more difficult, but were still able to perform on an equal level with adults. This is supported by the finding that caudate activation which increased progressively with age was also associated with better temporal discrimination. The

\section{REFERENCES}

Adleman, N. E., Menon, V., Blasey, C. M., White, C. D., Warsofsky, I. S., Glover, G. H., and Reiss, A. L. (2002). A developmental fMRI study of the stroop colour-word task. Neuroimage 16, 61-75.

Ardekani, B. A., Choi, S. J., HosseinZadeh, G. A., Porjesz, B., Tanabe, J. L., Lim, K. O., Bilder, R., Helpern, J. A., and Begleiter, H. (2002). Functional magnetic resonance imaging of brain activity in the visual oddball task. Brain Res. Cogn. Brain Res. 14, 347-356.

Blanton, R. E., Levitt, J. G., Peterson, J. R., Fadale, D., Sporty, M. L., Lee, M., To, D., Mormino, E. C., Thompson, P. M., Mccracken, J. T., and Toga, A. W. (2004). Gender differences in the left inferior frontal gyrus in normal children. Neuroimage 22, 626-636.

Block, R. A., Zakay, D., and Hancock, P. A. (1999). Developmental changes in human duration judgments: a meta-analytic review. Dev. Rev. 19, 183-211.

Booth, J. R., Burman, D. D., Meyer, J. R., Lei, Z., Trommer, B. L., Davenport, N. D., Li, W., Parrish, T. B., Gitelman, D. R., and Mesulam, M. (2003). Neural development of selective attention and response inhibition. Neuroimage 20, 737-751.

Braitenberg, V. (1967). Is the cerebellar cortex a biological clock in the millisecond range? Prog. Brain Res. 25, 334-346.

Brammer, M. J., Bullmore, E. T., and Simmons, A. (1997). Generic brain activation mapping in functional magnetic resonance imaging: a nonparametric approach. Magn. Reson. Imaging 15, 763-770.

Bullmore, E., Long, C., Suckling, J., Fadili, J., Calvert, G., Zelaya, F., Carpenter, T. A., and Brammer, M. (2001). Colored noise and computational inference in neurophysiological (fMRI) time series analysis: resampling methods in time and wavelet domains. Hum. Brain Mapp. 12, 61-78.

Bullmore, E. T., Brammer, M. J., and Rabe-Hesketh, S. (1999a). Methods for diagnosis and treatment of stimulus-correlated motion in generic brain activation studies using fMRI. Hum. Brain Mapp. 7, 38-48.

Bullmore, E. T., Suckling, J., Overmeyer, S., Rabe-Hesketh, S., Taylor, E., and Brammer, M. J. (1999b). Global, voxel, and cluster tests, by theory and permutation, for a difference between two groups of structural MR images of the brain. IEEE Trans. Med. Imaging 18, 32-42.

Bunge, S., Dudukovic, N., Thomason, M., Vaidya, C., and Gabrieli, J. (2002). Immature frontal lobe contributions to cognitive control in children: evidence from fMRI. Neuron 33, 301-311.

Christakou, A., Brammer, M., and Rubia, K. (2011). Maturation of limbic corticostriatal activation and connectivity associated with developmental changes in temporal discounting. Neuroimage 54, 1344-1354.

lack of performance differences is, in fact advantageous, as it shows that developmental changes are not an artifact of poorer performance in children, but reflect true activation differences between age groups.

To summarize, with increasing age, time discrimination is associated with progressively increased activation and interfunctional connectivity within later maturing left hemispheric lateral inferior and dorsolateral fronto-striato-thalamo-parietal networks that are known to be specialized for time discrimination, while earlier in life, there is greater involvement of structurally earlier developing ventromedial limbic, paralimbic, and cerebellar regions. The findings suggest there are age-dissociated neural networks of time discrimination with progressive left hemispheric inferior fronto-striato-parietal specialization with increasing maturation.

\section{ACKNOWLEDGMENTS}

This work was supported by grants from the Wellcome Trust (053272/Z/98/Z/JRS/JP/JAT), the Medical Research Council (GO300155), and PPP Healthcare (1206/1140). Dr. Smith and Dr. Simmons are supported by the NIHR Biomedical Research Centre for Mental Health at the South London and Maudsley NHS Foundation Trust and Institute of Psychiatry, Kings College London.

Christakou, A., Giampietro, V., Brammer, M., and Rubia, K. (2009a). Right ventromedial and dorsolateral prefrontal cortices mediate adaptive decisions under ambiguity by integrating choice utility and outcome evaluation. J. Neurosci. Methods 29, 11020-11028.

Christakou, A., Halari, R., Smith, A. B., Ifkovits, E., Brammer, M., and Rubia, K. (2009b). Sex-dependent age modulation of frontostriatal and temporo-parietal activation during cognitive control. Neuroimage 48, 223-236.

Chugani, H.T.,Phelps, M. E., and Mazziotta, J. C. (2008). "Positron emission tomography study of human brain functional development," in Brain Development and Cognition, eds M. Johnson, Y. Munakata, and R.O.Gilmore (NewYork: John Wiley and Sons),101-116.

Constantinidis, C., Williams, G. V. and Goldman-Rakic, P. S. (2002). A role for inhibition in shaping the temporal flow of information in prefrontal cortex. Nat. Neurosci. 5, 175-180.

Coull, J. T., Ruey-Kuang Cheng, R.K., and Meck, W. H. (2011). Neuroanatomical and neurochemical substrates of timing. Neuropsychopharmacology 36, 3-25.

Dawes, P., and Bishop, D. V. M. (2008). Maturation of visual and auditory temporal processing in school-aged children. J. Speech Lang. Hear. Res. 51, 1002-1015.

Devinsky, O., Morrell, M. J., and Vogt, B. A. (1995). Contributions of anterior cingulate cortex to behaviour. Brain 118, 279-306.

Drake, C., Jones, M. R., and Baruch, C. (2000). The development of rhythmic attending in auditory sequences: attunement, referent period, focal attending. Cognition 77, 251-288.

Droit-Volet, S., and Wearden, J. H. (2001).Temporal bisection in children. J. Exp. Child Psychol. 80, 142-159.

Dupont, P., Orban, G. A., Vogels, R., Bormans, G., Nuyts, J., Schiepers, C., De Roo, M., and Mortelmans, L. (1993). Different perceptual tasks performed with the same visual stimulus attribute activate different regions of the human brain: a positron emission tomography study. Proc. Natl. Acad. Sci. U.S.A.90, 10927-10931.

Ferrandez, A. M., Hugueville, L., Lehéricy, S., Poline, J. B., Marsault, C., and Pouthas, V. (2003). Basal ganglia and supplementary motor area subtend duration perception: an fMRI study. Neuroimage 19, 1532-1544.

Fischer, B., and Hartnegg, K. (2004). On the development of lowlevel auditory discrimination and deficits in dyslexia. Dyslexia 10, 105-118.

Floresco, S. B., Seamans, J. K., and Phillips, A. G. (1997). Selective roles for hippocampal, prefrontal cortical, and ventral striatal circuits in radial-arm maze tasks with or without a delay. J. Neurosci. 17, 1880-1890. 
Gautier, T., and Droit-Volet, S. (2002). Attentional distraction and time perception in children. Int. J. Psychol.37, 27-34.

Gogtay, N., Giedd, J., Lusk, L., Hayashi, K. M., Greenstein, D., Vaituzis, A. C., Nugent, T. F., Herman, D. H., Clasen, L., Toga, A. W., Rapoport, J. L., and Thompson, P. M. (2004). Dynamic mapping of human cortical development during childhood through early adulthood. Proc. Natl. Acad. Sci. U.S.A. 101, 8174-8179.

Greene, A. (1986). Future-time perspective in adolescence: the present of things future revisited. J. Youth Adolesc. 15, 99-113.

Grondin, S. (2010). Timing and time perception: a review of recent behavioral and neuroscience findings and theoretical directions. Atten. Percept. Psychophys. 72, 561-582.

Gruber, O., Kleinschmidt, A., Binkofski, F., Steinmetz, H., and Von Cramon, D. Y. (2000). Cerebral correlates of working memory for temporal information. Neuroreport 11, 1689-1693.

Gur, R. C., Turetsky, B. I., Loughead, J., Waxman, J., Snyder, W., Ragland, J. D., Elliott, M. A., Bilker, W., Arnold, S. E., and Gur, R. E. (2007). Hemodynamic responses in neural circuitries for detection of visual target and novelty: an event-related fMRI study. Hum. Brain Mapp. 28, 263-274.

Hale, S. (1990). A global developmental trend in cognitive processing speed. Child Dev. 61, 653-663.

Harrington, D. L., and Haaland, K. Y. (1999). Neural underpinnings of temporal processing: a review of focal lesion, pharmacological, and functional imaging research. Rev. Neurosci. 10, 91-116.

Ivry, R. B., and Diener, H. C. (1991). Impaired velocity perception in patients with lesions of the cerebellum. J. Cogn. Neurosci. 3, 355-366.

Ivry, R. B., and Hazeltine, R. E. (1995). Perception and production of temporal intervals across a range of durations: evidence for a common timing mechanism. J. Exp. Psychol. Hum. Percept. Perform. 21, 3-18.

Ivry, R. B., and Keele, S. W. (1988). Dissociation of the lateral and medial cerebellum in movement timing and movement execution. Exp. Brain Res. 73, 136-152.

Joseph, R. (1999). Environmental influences on neural plasticity, the limbic system, emotional development and attachment: a review. Child Psychiatry Hum. Dev. 29, 189-208.

Jueptner, M., Rijntjes, M., Weiller, C., Faiss, J. H., Timmann, D., Mueller,
S. P., and Diener, H. C. (1995). Localization of a cerebellar timing process using PET. Neurology 45, 1540-1545.

Justus, R. B., and Ivry, T. (2001). The cognitive neuropsychology of the cerebellum. Int. Rev. Psychiatry 13, 276-282.

Keele, S., Polorny, R., Corcos, D., and Ivry, R. (1985). Do perception and motor production share common timing mechanisms: a correlational analysis. Acta Psychol. (Amst) 60.

Kiehl, K. A., Laurens, K. R., Duty, T. L., Forster, B. B., and Liddle, P. F. (2001). An event-related fMRI study of visual and auditory oddball tasks. J. Psychophysiol. 15, 221-240.

Konrad, K., Neufang, S., Thiel, C. M., Specht, K., Hanisch, C., Fan, J., Herpertz-Dahlmann, B., and Fink, G. R. (2005). Development of attentional networks: an fMRI study with children and adults. Neuroimage 28, 429-439.

Kowal, S., O'connell, D. C., and Sabin, E. J. (1975). Development of temporal patterning and vocal hesitations in spontaneous narratives. $J$. Psycholinguist. Res. 4, 195-207.

Lark, V. P., Fannon, S., Lai, S., Benson, R., and Bauer, L. (2000). Responses to rare visual target and distractor stimuli using event-related fMRI. J. Neurophysiol. 83, 3133-3139.

Lawrence, N., Jollant, F. O. O. D., Zelaya, F., and Phillips, M. (2009). Distinct roles of prefrontal cortical subregions in the Iowa Gambling Task. Cereb. Cortex 19, 1134-1143.

Lejeune, H., Maquet, P., Pouthas, P., Bonnet, M., Casini, L., Macar, F., Vidal, F., Ferrara, A., and TimsitBerthier, M. (1997). Brain activation correlates of synchronization: a PET study. Neurosci. Lett. 235, 21-24.

Lewis, P. A. (2002). Finding the timer. Trends Cogn. Sci. (Regul. Ed.) 6, 195-196.

Livesey, A. C., Wall, M. B., and Smith, A. T. (2007). Time perception: manipulation of task difficulty dissociates clock functions from other cognitive demands. Neuropsychologia 45, 321-331.

Luna, B., Thulborn, K. R., Munoz, D. P., Merriam, E. P., Garver, K. E., Minshew, N. J., Keshavan, M. S., Genovese, C. R., Eddy, W. F., and Sweeney, J. A. (2001). Maturation of widely distributed brain function subserves cognitive development. Neuroimage 13, 786-793.

Macar, F., Coull, J. T., and Vidal, F. (2006). The supplementary motor area in motor and perceptual time processing: fMRI studies. Cogn. Process. 7, 89-94.
Mangels, J. A., Ivry, R. B., and Shimizu, N. (1998). Dissociable contributions of the prefrontal and neocerebellar cortex to time perception. Brain Res. Cogn. Brain Res. 7 , 15-39.

Maquet, P., Lejeune, H., Pouthas, V., Bonnet, M., Casini, L., Macar, F. Timsit-Berthier, M., Vidal, F., Ferrara, A., Degueldre, C., Quaglia, L., Delfiore, G., Luxen, A., Woods, R., Mazziotta, J. C., and Comar, D. (1996). Brain activation induced by estimation of duration: a PET study. Neuroimage 3, 119-126.

Marsh, R., Zhu, H., Schultz, R. T., Quackenbush, G., Royal, J., Skudlarski, P., and Peterson, B. S. (2006). A developmental fMRI study of self-regulatory control. Hum. Brain Mapp. 27, 848-863.

Matell, M. S., and Meck, W. H. (2004). Cortico-striatal circuits and interval timing: coincidence detection of oscillatory processes. Brain Res. Cogn. Brain Res. 21, 139-170.

Mathiak, K., Hertrich, I., Grodd, W., and Ackermann, H. (2004). Discrimination of temporal information at the cerebellum: functional magnetic resonance imaging of nonverbal auditory memory. Neuroimage 21, 154-162.

McAuley, J., Riess-Jones, M., Holub, S., Johnston, H. M., and Miller, N. S. (2006). The time of our lives: life span development of timing and event tracking. J. Exp. Psychol. Gen. 135, 348-367.

Mesulam, M. M., Nobre, A. C., Kim, Y. H., Parrish, T. B., and Gitelman, D. R. (2001). Heterogeneity of cingulate contributions to spatial attention. Neuroimage 13, 1065-1072.

Mohanty, A., Gitelman, D. R., Small, D. M., and Mesulam, M. M. (2008). The spatial attention network interacts with limbic and monoaminergic systems to modulate motivationinduced attention shifts. Cereb. Cortex 18, 2604-2613.

Monk, C. S., Mcclure, E. B., Nelson, E. E., Zarahn, E., Bilder, R. M., Leibenluft, E., Charney, D. S., Ernst, M., and Pine, D. S. (2003). Adolescent immaturity in attentionrelated brain engagement to emotional facial expressions. Neuroimage 20, 420-428.

Morillon, B., Kell, C. A., and Giraud, A.-L. (2009). Three stages and four neural systems in time estimation. $J$. Neurosci. 29, 14803-14811.

Nenadic, I., Gaser, C., Volz, H.-P., Rammsayer, T., Hager, F., and Sauer, H. (2003). Processing of temporal information and the basal ganglia: new evidence from fMRI. Exp. Brain Res. 148, 238-246.

Neufang, S., Fink, G. R., HerpertzDahlmann, B., Willmes, K., and Konrad, K. (2008). Developmental changes in neural activation and psychophysiological interaction patterns of brain regions associated with interference control and time perception. Neuroimage 43, 399-409.

Nichols, T., Brett, M., Andersson, J., Wager, T., and Poline, J.-B. (2005). Valid conjunction inference with the minimum statistic. Neuroimage 25, 653-660.

Ortuno, F., Ojeda, N., Arbizu, J., Lopez, P., Marti-Climent, J. M., Penuelas, I., and Cervera, S. (2002). Sustained attention in a counting task: normal performance and functional neuroanatomy. Neuroimage 17, 411-420.

Pardo, J. V., Fox, P. T., and Raichle, M. E. (1991). Localisation of a human system for sustained attention by positron emission tomography. Nature 349, 61-64.

Paus, T., Petrides, M., Evans, A. C., and Meyer, E. (1993). Role of the human anterior cingulate cortex in the control of oculomotor, manual, and speech responses: a positron emission tomography study. J. Neurophysiol. 70, 453-469.

Pedersen, C. B., Mirz, F., Ovesen, T., Ishizu, K., Johannsen, P., Madsen, S., and Gjedde, A. (2000). Cortical centres underlying auditory temporal processing in humans: a PET study. Audiology 39, 30-37.

Penhune, V. B., Zatorre, R. J., and Evans, A. C. (1998). Cerebellar contributions to motor timing: a PET study of auditory and visual rhythm reproduction. J. Cogn. Neurosci. 10, 752-765.

Rao, S. M., Harrington, D. L., Haaland, K. Y., Bobholz, J. A., Cox, R. W., and Binder, J. R. (1997). Distributed neural systems underlying the timing of movements. J. Neurosci. 17, 5528-5535.

Rao, S. M., Mayer, A. R., and Harrington, D. L. (2001). The evolution of brain activation during temporal processing. Nat. Neurosci. 4, 317-323.

Raven, J., Raven, J. C., and Court, J. H. (1998). Manual for Raven's Progressive Matrices and Vocabulary Scales, Section 3: The Standard Progressive Matrices. San Antonio, TX: The Psychological Corporation.

Ridderinkhof, K. R., Ullsperger, M., Crone, E. A., and Nieuwenhuiss, S. (2004). The role of the medial frontal cortex in cognitive control. Science 306, 443-447. 
Rogers, R. D., Ramnani, N., Mackay, C., Wilson, J. L., Jezzard, P., Carter, C. S., and Smith, S. M. (2004). Distinct portions of anterior cingulate cortex and medial prefrontal cortex are activated by reward processing in separable phases of decisionmaking cognition. Biol. Psychiatry 55, 594-602.

Rozek, F.,Wessman, A. E., and Gorman, B. S. (1977). Temporal span and delay of gratification as a function of age and cognitive development. J. Genet. Psychol. 131, 37-40.

Rubia, K. (2006). "The neural correlates of timing functions," in Timing the Future: The Case for a Time-Based Prospective Memory, eds J. Glicksohn and M. S. Myslobodsky (New Jersey: World Scientific Publishing), 213-238.

Rubia, K., Hyde, Z., Halari, R., Giampietro, V., and Smith, A. (2010). Effects of age and sex on developmental neural networks of visual-spatial attention allocation. Neuroimage 51, 817-827.

Rubia, K., Overmeyer, S., Taylor, E., Brammer, M., Williams, S., Simmons, A., Andrew, C., and Bullmore, E. (2000). Functional frontalisation with age: mapping neurodevelopmental trajectories with fMRI. Neurosci. Biobehav. Rev. 24, 13-19.

Rubia, K., and Smith, A. (2004). The neural correlates of cognitive time management: a review. Acta Neurobiol. Exp. (Wars) 64, 329-340.

Rubia, K., Smith, A., Brammer, M., and Taylor, E. (2003). Right inferior prefrontal cortex mediates response inhibition while mesial prefrontal cortex is responsible for error detection. Neuroimage 20, 351-358.

Rubia, K., Smith, A., Taylor, E., and Brammer, M. (2007). Linear agecorrelated functional development of right inferior fronto-striatocerebellar networks during response inhibition and anterior cingulate during error-related processes. Hum. Brain Mapp. 28, 1163-1177.

Rubia, K., Smith, A. B., Woolley, J., Nosarti, C., Heyman, I., Brammer, M., and Taylor, E. (2006). Progressive increase of frontostriatal brain activation from childhood to adulthood during event-related tasks of cognitive control. Hum. Brain Mapp. 27, 973-993.

Rueckriegel, S. M., Blankenburg, F. Burghardt, R., Ehrlich, S., Henze, G. N., Mergl, R., and Hernáiz Driever, P. (2008). Influence of age and movement complexity on kinematic hand movement parameters in childhood and adolescence. Int. J. Dev. Neurosci. 26, 655-663.

Schoenbaum, G., Roesch, M. R., and Stalnaker, T. A. (2006). Orbitofrontal cortex, decision-making and drug addiction. Trends Neurosci. 29, 116-124.

Seeley, W. W., Menon, V., Schatzberg, A. F., Keller, J., Glover, G. H., Kenna, H., Reiss, A. L., and Greicius, M. D. (2007). Dissociable intrinsic connectivity networks for salience processing and executive control. J. Neurosci. 27, 2349-2356.

Shih, L. Y. L., Kuo, W.-J., Yeh, T.C., Tzeng, O. J. L., and Hsieh, J. C. (2009). Common neural mechanisms for explicit timing in the sub-second range. Neuroreport 20, 897-901.

Simmons, A., Moore, E., and Williams, S. C. R. (1999). Quality control for functional MRI using automated data analysis and Shewhart charting. Magn. Res. Imaging Med. 41, 1274-1278.

Small, D. M., Gitelman, D. R., Gregory, M. D., Nobre, A. C., Parrish, T. B., and Mesulam, M. M. (2003). The posterior cingulate and medial prefrontal cortex mediate the anticipatory allocation of spatial attention. Neuroimage 18, 633-641.

Smith, A., Halari, R., Giampietro, V., Brammer, M., and Rubia, K. (2011).
Developmental effects of reward on sustained attention networks. Neuroimage 56, 1694-1704.

Smith, A. B., Taylor, E., Lidzba, K. and Rubia, K. (2003). A right hemispheric frontocerebellar network for time discrimination of several hundreds of milliseconds. Neuroimage 20, 344-350.

Smith, S. M. (2002). Fast robust automated brain extraction. Hum. Brain Mapp. 17, 143-155.

Sowell, E. R., Peterson, B. S., Thompson, P. M., Welcome, S. E., Henkenius, A. L., and Toga, A. W. (2003). Mapping cortical change across the human life span. Nat. Neurosci. 6 , 309-315.

Sowell, E. R., Thompson, P. M., Holmes, C. J., Jernigan, T. L., and Toga, A. W. (1999). In vivo evidence for post-adolescent brain maturation in frontal and striatal regions. Nat. Neurosci. 2, 859-861.

Steinberg, L., Graham, S., O'brien, L., Woolard, J., Cauffman, E., and Banich, M. T. (2009). Age differences in future orientation and delay discounting. Child Dev. 80, 28-44.

Stevens, M. C., Kiehl, K. A., Pearlson, G. D., and Calhoun, V. D. (2007). Functional neural networks underlying response inhibition in adolescents and adults. Behav. Brain Res 181, 12-22.

Szelag, E., Kowalska, J., Rymarczyk, K. and Pöppel, E. (2002). Duration processing in children as determined by time reproduction: implications for a few seconds temporal window. Acta Psychol. 110, 1-19.

Tamm, L., Menon, V., and Reiss, A. L. (2002). Maturation of brain function associated with response inhibition. J. Am. Acad. Child Adolesc. Psychiatry 41, 1232-1238.

Thirion, B., Pinel, P., Meriaux, S. Roche, A., Dehaene, S., and Poline, J.-B. (2007). Analysis of a large fMRI cohort: statistical and methodological issues for group analysis. Neuroimage 35 , 105-120.

Tregellas, J. R., Davalos, D. B., and Rojas, D. C. (2006). Effect of task difficulty on the functional anatomy of temporal processing. Neuroimage 32, 307-315.

Ullsperger, M., and van Cramon, D. Y. (2001). Subprocesses of performance monitoring: a dissociation of error processing and response competition revealed by event-related fMRI and ERPs. Neuroimage 14, 1387-1401.

Wittmann, M., Simmons, A. N., Aron, J. L., and Paulus, M. P. (2010). Accumulation of neural activity in the posterior insula encodes the passage of time. Neuropsychologia 48, 3110-3120.

Zhang, H., Nichols, T. E., and Johnson, T. D. (2009). Cluster mass inference via random field theory. Neuroimage 44, 51-61.

Conflict of Interest Statement: The authors declare that the research was conducted in the absence of any commercial or financial relationships that could be construed as a potential conflict of interest.

Received: 14 July 2011; accepted: 26 October 2011; published online: 11 November 2011.

Citation: Smith AB, Giampietro $V$, Brammer $M$, Halari $R$, Simmons $A$ and Rubia $K$ (2011) Functional development of fronto-striato-parietal networks associated with time perception. Front. Hum. Neurosci. 5:136. doi: 10.3389/fnhum.2011.00136

Copyright (C) 2011 Smith, Giampietro, Brammer, Halari, Simmons and Rubia. This is an open-access article subject to a non-exclusive license between the authors and Frontiers Media SA, which permits use, distribution and reproduction in other forums, provided the original authors and source are credited and other Frontiers conditions are complied with. 